\title{
Avaliação de progressão da competência de estudantes de medicina na realização de pontos cirúrgicos em simulador de laparoscopia
}

\section{Evaluation of progression of competence in performing surgical points in laparoscopic simulator by medical students}

\author{
Grijalva Otávio Ferreira da Costa $^{1,2}$ [D, Hermano Alexandre Lima Rocha ${ }^{3}\left(\mathbb{C}\right.$, Luiz Gonzaga de Moura Junior ${ }^{4}$, Francisco das \\ Chagas Medeiros ${ }^{4}(\mathbb{0}$
}

1. Docente do curso de Medicina do Centro Universitário Christus (UNICHRISTUS), Fortaleza, CE, Brasil. 2. Doutorando em Ciências Médico-Cirúrgicas pela Universidade Federal do Ceará (UFC), Fortaleza, CE, Brasil. 3. Docente do Programa de Pós-graduação em Saúde Coletiva da Universidade Federal do Ceará (UFC), Fortaleza, CE, Brasil. 4. Docente do curso de Medicina pela Universidade Federal do Ceará (UFC), Fortaleza, CE, Brasil. 5. Departamento de Saúde Materno-Infantil, Membro do Programa de pós-graduação de ciências médico-cirurgicas, Faculdade de Medicina, Universidade Federal do Ceará

\begin{abstract}
Resumo
Objetivo: avaliar a progressão da competência e a curva de aprendizagem de estudantes de Medicina submetidos a treinamento para a realização de nós e pontos cirúrgicos. Métodos: estudo prospectivo, longitudinal, intervencionista, realizado de abril/2016 a janeiro/2017, com a participação de 29 estudantes de Medicina, do primeiro ao terceiro ano, com progressão de habilidades para realização de nós e pontos laparoscópicos em ambiente de simulação em quatro etapas, com duração de 16 horas. Foi estabelecida a tarefa de realizar pontos laparoscópicos, com cinco seminós, em molde de sutura, em 18 minutos. Foram realizados os testes de ANOVA, Mann-Whitney e o qui-quadrado. Foram realizadas regressões lineares simples e múltiplas, sendo consideradas significativas as comparações com valor de $\mathrm{p} \leq 0,05$. Resultados: foram analisadas as médias do número de seminós $(1,0$ a 14,0 , $\beta($ IC-95\%) 4,49 (3,80/5,19), pontos laparoscópicos intracorpóreos $(0,1$ a 2,7, $\beta($ IC-95\%) $0,87(0,72 / 1,02)$, os tamanhos dos cotos dos fios $(0,1$ a 2,9, $\beta($ IC-95\%) 0,95 $(0,72 / 1,18)$ e ajustes dos seminós iniciais $(0,2$ a $2,9, \beta($ IC-95\%) $0,92(0,76 / 1,07)$ e sequenciais $(0,3$ a $10,0, \beta($ IC-95\%) 3,32 (2,84/3,80), entre as etapas do treinamento. Houve significância estatística em todos os parâmetros avaliados. A progressão na execução de pontos laparoscópicos foi evidente com coeficiente de regressão linear igual a 0,511. A curva de aprendizagem demonstrou que $96,3 \%$ dos estudantes realizaram, pelo menos, um ponto laparoscópico ao final do treinamento. Conclusão: o estudo revelou que os alunos apresentaram progressão na competência e evolução na curva de aprendizagem na realização de pontos laparoscópicos em ambiente de simulação ao final do treinamento.
\end{abstract}

Palavras-chave: Cirurgia laparoscópica. Treinamento por simulação. Educação médica. Habilidade cirúrgica.

\begin{abstract}
Objective: to evaluate the progression of competency and the learning curve of medical students undergoing practical, systematic, methodical training for performing knots and instrumental points in a video-surgery simulation environment. Methods: a prospective, longitudinal, interventional study was carried out between April 2016 and January 2017 with the participation of 29 medical students from the Christus University Center, Fortaleza, Brazil, from the first to the third year, subjected to practical theoretical training, systematized, with progression of skills for realization of laparoscopic points in simulation environment in four stages during 16 hours. A standardized task was established to perform laparoscopic points, with five throws, in suture mold, abdominal cavity simulator, in 18 minutes. The students were evaluated at all stages of the training. ANOVA or Mann-Whitney tests were performed for numerical variables, and the chi-square test was performed for categorical variables. Simple and multiple linear regressions were performed, and $\mathrm{p} \leq$ 0.05 was considered significant. Results: the mean number of throws $(1.0$ to $14.0, \beta(95 \% \mathrm{CI}), 4.49(3.80 / 5.19)$ and laparoscopic points $(0.1$ to 2.7 , $\beta$ ( $95 \%$ CI) 0.87 ( 0.72 / 1.02) performed within 18 minutes, thread lengths $(0.1$ to $2.9, \beta(95 \% \mathrm{CI})(0.72 / 1.18)$ and the adjustments of the initial throws ( 0.2 to $2.9, \beta(95 \% \mathrm{CI}) 0.92(0.76 / 1.07)$ and sequential throws $(0.3(95 \% \mathrm{CI})$ yielded a mean test value of $10.0, \beta(95 \% \mathrm{CI}) 3.32(2.84$ / 3.80$)$ across the various stages of the training. The progression in execution of laparoscopic points was evident, with linear regression coefficient equal to 0.511 . The learning curve showed that $96.3 \%$ of the students performed at least one laparoscopic point in the fourth stage of training. Conclusion: the study showed that students presented progression in competence and evolution along the learning curve in the accomplishment of laparoscopic points in simulation environment at the end of training.
\end{abstract}

Key words: Laparoscopic surgery. Simulation-based learning. Medical education. Surgical skills.

\section{INTRODUÇÃO}

O ensino de procedimentos cirúrgicos tem sido, historicamente, realizado com treinamento em pacientes, em situações reais, supervisionados no campo operatório por um cirurgião experiente. Essa prática foi adotada desde a criação dos programas de residência médica e ainda permanece como padrão até os dias atuais. Como consequência, a curva de aprendizagem de alguns procedimentos é, usualmente, longa, e algumas iatrogenias podem, eventualmente, acontecer ${ }^{1}$.

O advento de novas tecnologias tem obrigado aos médicos, de 
uma maneira geral e, em particular, aos cirurgiões a adquirir novas e desafiadoras habilidades.

A partir da década de 1980, com o desenvolvimento da laparoscopia, novas habilidades e competências tiveram que ser adquiridas por cirurgiões e residentes de especialidades cirúrgicas em todo o mundo. Entre essas habilidades, a realização de nós, pontos e suturas são inegavelmente, os procedimentos mais difíceis e complexos ${ }^{2}$.

Com a disseminação do método laparoscópico na prática clínica e sua introdução como técnica cirúrgica a ser aprendida nos programas de residência médica, foram estabelecidos treinamentos com o uso de simuladores para acelerar o processo do saber fazer e diminuir o risco do treinamento em pacientes ${ }^{3}$.

Classicamente, a grande dificuldade na capacitação em procedimentos laparoscópicos é a aquisição da competência para trabalhar em duas dimensões com a visão direcionada para o monitor e a manipulação do instrumental sem visão direta, percepção de profundidade alterada, campo de trabalho reduzido, posição fixa dos trocartes, além da percepção de inversão dos movimentos das pinças (efeito fulcral) e perda da percepção háptica ${ }^{4}$ devido à resistência dentro dos trocartes e aos instrumentos cirúrgicos longos ${ }^{5}$. Essas particularidades fazem que a capacitação na técnica laparoscópica apresente singularidades e abordagens próprias ${ }^{6}$.

Em contraste com a laparoscopia, a capacitação nas técnicas operatórias "a céu aberto", no qual o trabalho se processa sob a visão direta, e a incorporação pelo cirurgião das habilidades psicomotoras são, proporcionalmente, mais fáceis ${ }^{7}$.

Ao longo dos anos 1990 e nas primeiras décadas do século XXI, publicações demonstraram que o treinamento em simuladores diminuía o tempo de aprendizagem dos procedimentos laparoscópicos, e a sua utilização começou a ser padronizada nos serviços de formação profissional ${ }^{8,9,10}$.

Várias pesquisas sugeriam que tarefas realizadas no laboratório de simulação poderiam ajudar na habilidade cirúrgica do residente com muita confiabilidade ${ }^{11,12}$. O treinamento com simulação, usando instrumentos reais e equipamentos de laparoscopia, constituiu-se, então, em uma nova estratégia de capacitação de cirurgiões ${ }^{13}$.

Naturalmente, diversos programas de capacitação nos quais se usavam simuladores em procedimentos laparoscópicos foram criados e ofertados a residentes de cirurgia, cirurgiões e especialistas da área. A maioria estabelecia o processo do saber fazer sem a garantia de uma capacitação previamente testada e quase sempre baseada na vivência de um expert nos procedimentos $^{14,15}$.

Dessa forma, o laboratório de simulação cirúrgica tornou-se importante e até indispensável na evolução da laparoscopia, sendo usado em capacitação, ensino, testes de técnicas cirúrgicas inovadoras e desenvolvimento de instrumentais e equipamentos ${ }^{16}$.

No Brasil, parece haver um consenso de que a capacitação para aquisição de habilidades em laparoscopia necessita de processo pedagógico mais adequado de ensino nas residências médicas para conferir uma base educacional mais sólida que a atual ${ }^{17}$.

Recentemente, novas estratégias de capacitação para médico estão surgindo, e estudos têm demonstrado a correlação entre a habilidade de visão espacial e a memória do trabalho visual no desempenho com simuladores cirúrgicos. Tal fato, quando utilizado, deverá melhorar o desempenho dos cirurgiões ${ }^{18}$.

Assim como na capacitação de cirurgiões, a dos médicos residentes em técnicas de laparoscópica também é baseada, na maioria dos programas, em modelo de ensino cientificamente não testado e sem a garantia de competência no processo do saber fazer ao final do treinamento ${ }^{13}$.

Desse modo, o presente estudo foi realizado com os objetivos de avaliar a progressão da competência e a curva de aprendizagem dos estudantes de Medicina submetidos a treinamento teórico-prático, sistematizado, metodizado, com progressão de habilidades na realização de nós e pontos manuais e instrumentais, em ambiente de simulação de laparoscopia.

\section{MÉTODOS}

Trata-se de um estudo prospectivo, longitudinal, intervencionista com o objetivo de avaliar a progressão da competência de estudantes de Medicina submetidos a um modelo teóricoprático de ensino na realização de nós e pontos cirúrgicos em simulador de laparoscopia.

\section{Grupo de estudo}

Foram acompanhados 29 estudantes que cursavam até o terceiro ano do Curso de Medicina no Centro Universitário Christus, Unichristus, na cidade de Fortaleza, Ceará. A pesquisa foi realizada no período de abril de 2016 a janeiro de 2017, no laboratório de habilidades cirúrgicas do Centro Universitário Christus, em quatro turnos de quatro horas de duração, totalizando 16 horas de treinamento/capacitação. Em cada treinamento, foram aceitos, no máximo, oito participantes. (Figura 1)

Os dados demográficos dos estudantes foram coletados no momento da inscrição para realização do treinamento.

Foram avaliados os seguintes dados: idade, sexo, mão dominante, habilidade em digitação em celulares e teclados de computação, vivências prévias em cirurgia convencional e laparoscópica, prática de instrumentos musicais e ou videogames e o desejo de exercer, no futuro, atividades cirúrgicas.

\section{Critérios de inclusão e exclusão}

Como critérios de inclusão, os alunos poderiam ser de ambos os sexos, maiores de 18 anos. Foram excluídos da análise 
aqueles que, por qualquer motivo, desistiram do treinamento em qualquer momento e os que não apresentaram limitações de ordem anatômica e/ou funcional para o desempenho das tarefas propostas.

Figura 1. A-Laboratório de habilidades cirúrgica do Centro Universitário Christus. B-Simulador EndoSuture Training Box ${ }^{\circledR}$, instrumentais. C-Placa de sutura sintética em formato de estômago humano.

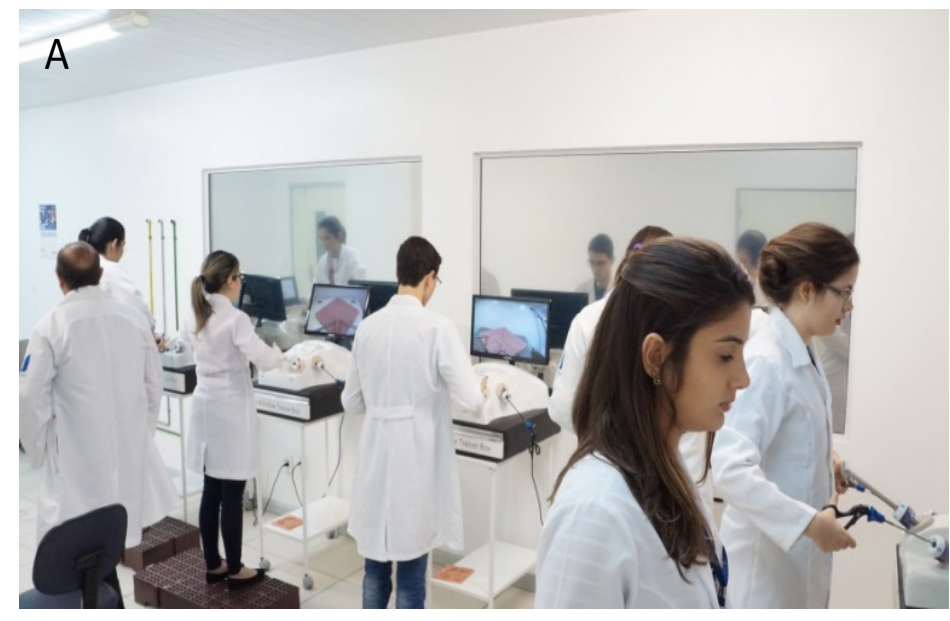

Modelo de treinamento

A capacitação foi composta de um modelo de treinamento teórico-prático de ensino realizado em quatro etapas, dispostas em graus progressivos de dificuldade para facilitar a obtenção da competência. A primeira e a segunda etapas tiveram como objetivo o treinamento para o desenvolvimento de habilidades em realizar nós e pontos cirúrgicos manuais e instrumentais convencionais. A terceira e quarta etapas objetivaram a adaptação ao ambiente simulado de laparoscopia e treinamento para o desenvolvimento de habilidades em realizar nós e pontos laparoscópicos em três e duas dimensões, respectivamente.

Foram utilizadas para fundamentação do modelo teóricoprático de ensino a Taxonomia de Dave e as Teorias de Fitts e Posner, Ericsons, Miller e a ferramenta do feedback ${ }^{19,20}$.

Para avaliar a progressão da competência na realização de nós e pontos laparoscópicos, foram realizados testes no início do treinamento (etapa 1) e, ao final, das etapas 2, 3 e 4 . No início de cada teste, os alunos foram orientados a realizar o maior número de pontos laparoscópicos ajustados cada um com cinco seminós ${ }^{21,22}$, em placa de sutura sintética utilizando um fio sutura de seda 2.0 agulhado cilíndrico de $2,5 \mathrm{~cm}$ e $3 / 8$ de curvatura, respeitando as marcações prévias no molde de sutura.

Optou-se por esse tipo de nó por ser seguro e de maior resistência ao fenômeno do deslizamento ${ }^{23}$. Os nós não deveriam apresentar espaço entre os seminós iniciais e sequenciais e as pontas dos fios seccionadas ter entre 5 e 7 milímetros. 0 tempo de execução da tarefa foi limitado a 18 minutos.

Para execução do teste, os estudantes dispuseram de simulador de ambiente laparoscópico (EndoSuture Training Box ${ }^{\circledR}$ ), um porta-agulhas, uma pinça de apreensão e uma tesoura todos
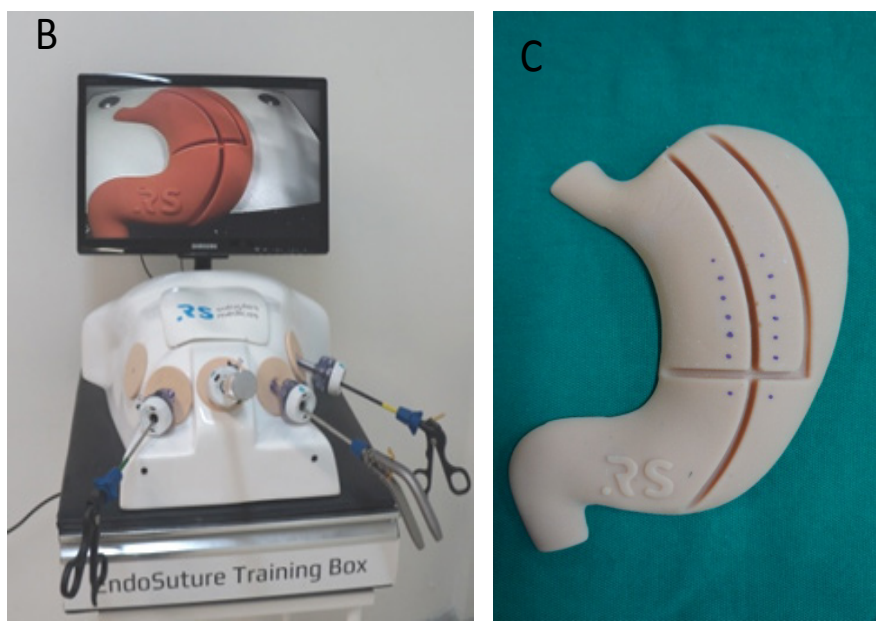

para uso laparoscópico, além de fio de sutura de seda 2.0 de $22 \mathrm{~cm}$ de comprimento, com agulha cilíndrica de $2,5 \mathrm{~cm}$ e uma placa de sutura sintética de elastômero termoplástico em formato de estômago humano com sulcos para realização dos nós e pontos cirúrgicos (Figura 1).

\section{Critérios de avaliação}

A avaliação da progressão da competência considerou os seguintes parâmetros: número de seminós e pontos realizados em 18 minutos, obediência à marcação prévia dos pontos, tamanho dos cotos dos fios cirúrgicos entre 5 e 7 milímetros, ajuste do seminó inicial e ajuste dos seminós sequenciais, sendo considerados adequados aqueles que não apresentassem espaços entre os seminós ao exame a olho nu.

Foram, também, avaliados os números totais de obediência e não obediência à marcação prévia dos pontos, de cotos de fios adequados e não adequados e dos seminós iniciais e sequenciais ajustados e não ajustados, além das curvas de aprendizagem dos estudantes. Os testes, em cada uma das etapas, foram feitos, simultaneamente, para todos os participantes.

A coleta de dados ocorreu no início da etapa 1 (avaliação inicial) e, ao final das demais etapas, em formulário próprio. Todos os testes executados foram fotografados e arquivados, com autorização dos participantes.

\section{Análise estatística}

Os resultados quantitativos categóricos foram apresentados em forma de percentuais e as variáveis numéricas utilizando as medidas de tendência central. Testes de normalidade para variáveis numéricas foram realizados, assim como regressões lineares simples e múltiplas para verificar a significância estatística das correlações, sendo consideradas significativas as 
comparações com valor de $p<0,05$. Para construção das curvas de aprendizagem, foram utilizados modelos regressivos de Cox. Os dados foram tabulados e analisados, utilizando o software SPSS (Statistical Package for the Social Sciences), v23, SPSS, Inc.

A avaliação da competência dos alunos na execução das tarefas propostas de acordo com as etapas do treinamento foi analisada, utilizando-se a regressão linear entre o número de avaliações e as médias de pontuações obtidas.

\section{Aspectos éticos}

Todos os participantes do estudo foram esclarecidos sobre a pesquisa. A pesquisa foi submetida e aprovada pelo Comitê de Ética e Pesquisa do Centro Universitário Christus, sob o n으 51295815.5.0000.5049-21/12/2015.

\section{RESULTADOS}

A média das idades dos estudantes foi de 21,1 anos, sendo 17 $(58,6 \%)$ do sexo feminino e $12(41,4 \%)$ do sexo masculino. Vinte e seis estudantes $(89,7 \%)$ apresentavam domínio na mão direita e $7(24,1 \%)$ praticavam videogame três ou mais horas por semana. Vinte e dois $(75,9 \%)$ referiram que não tinham vivência ou qualquer experiência em cirurgia convencional, enquanto vinte e seis $(89,7 \%)$ referiram o mesmo em relação à videocirurgia. Foi considerada vivência em cirurgia ou videocirurgia o fato de a ter presenciado ou participado em campo operatório de qualquer procedimento. Vinte e oito $(96,6 \%)$ consideravam-se hábeis na digitação de teclados de computadores ou similares, e vinte e sete desejavam, no futuro, exercer profissionalmente atividades cirúrgicas $(93,1 \%)$ (tabela 1$)$.

Tabela 1. Distribuição dos dados demográficos dos estudantes de Medicina em média/desvio padrão e números absolutos e percentuais.

\begin{tabular}{lrrr}
\hline Dados demográficos & & Média/n & DP/\% \\
\hline Idade & anos & 21,1 & 2,0 \\
Sexo & feminino & 17 & $58,6 \%$ \\
& masculino & 12 & $41,4 \%$ \\
Mão dominante & direita & 26 & $89,7 \%$ \\
& esquerda & 3 & $10,3 \%$ \\
Hábil em digitar no celular & & 28 & $96,6 \%$ \\
Hábil em digitar teclados de computação & & 27 & $93,1 \%$ \\
Desejo de exercer atividades cirúrgicas & & 27 & $93,1 \%$ \\
Prática de instrumento musical & & 8 & $27,6 \%$ \\
Vivência em cirurgia & & 7 & $24,1 \%$ \\
Prática de videogame três ou mais h/ & & 7 & $24,1 \%$ \\
semana. & & 3 & $10,3 \%$ \\
Vivência em videocirurgia & & & \\
\hline DP: Desvio padrão & &
\end{tabular}

Os valores das médias dos critérios de avaliação dos nós e pontos laparoscópicos executados apresentaram progressão entre todas as etapas do modelo de treinamento, sendo mais acentuado entre a terceira e a quarta etapas, em todos os itens avaliados.

As médias das obediências às marcações prévias dos pontos variaram de 0,7 a 5,8 entre as etapas de treinamento. As médias do número de cotos dos fios adequados variaram de 0,1 a 2,9 entre a 1a e 4a etapas. As médias dos seminós iniciais e sequenciais ajustados variaram, respectivamente, entre as etapas de 0,2 a 2,9 e de 0,3 a 10,0. Todas as tendências foram, estatisticamente, significantes com valores de $p$ menores que 0,001 (Tabela 2). Na quarta avaliação, um aluno executou seis pontos ajustados laparoscópicos. Os demais realizaram entre 1 e 5 pontos, e somente um não executou nenhum ponto por completo.

A comparação entre as medianas da primeira etapa (avaliação inicial) e a quarta etapa (avaliação final) de todos os critérios de avaliação dos nós e pontos laparoscópicos apresentaram nível de significância com valores de $p<0,001$, o que demonstra, estatisticamente, a progressão da competência dos estudantes na realização da tarefa proposta em ambiente de simulação. Foi utilizado nessa análise o teste ANOVA de medidas repetidas (tabela 2).

A progressão na execução de pontos laparoscópicos foi evidente entre as etapas do treinamento com coeficiente de regressão linear igual a 0,511 (Figura 2), assim como a curva de aprendizagem, a qual demonstra que 96,3\% dos estudantes realizaram, pelo menos, um ponto laparoscópico ao final do treinamento.

A progressão da habilidade e a curva de aprendizagem foram mais acentuadas nas terceiras e quartas etapas do treinamento (Figura 3). 
Tabela 2. Médias das avaliações, desvios padrões, beta e níveis de significâncias dos critérios de avaliação na execução de nós e pontos laparoscópicos em simulador de laparoscopia.

\begin{tabular}{lrrrrrr}
\hline \multicolumn{1}{c}{$\mathbf{n}$} & Etapa 1 & Etapa 2 & Etapa 3 & Etapa 4 & & \\
& $-\mathbf{x}(\mathbf{D P})$ & $-\mathbf{( x ) ( D P )}$ & $-\mathbf{x ( D P )}$ & $-\mathbf{x}(\mathbf{D P})$ & $\boldsymbol{\beta}($ IC-95\%) & $\mathbf{p}$ \\
\hline Seminós em 18 minutos & $1,0(1,5)$ & $3,2(2,7)$ & $9,0(4,6)$ & $14,0(6,2)$ & $4,49(3,80 / 5,19)$ & 0,001 \\
Pontos em 18 minutos & $0,1(0,4)$ & $0,4(0,6)$ & $1,5(1,0)$ & $2,7(1,3)$ & $0,87(0,72 / 1,02)$ & 0,001 \\
Obediência à marcação & $0,7(1,0)$ & $1,3(1,1)$ & $3,1(2,1)$ & $5,8(2,9)$ & $1,70(1,37 / 2,02)$ & 0,001 \\
Total obed. e não obed. & $1,5(1,2)$ & $2,3(1,1)$ & $4,3(1,8)$ & $6,6(2,5)$ & $1,73(1,43 / 2,02)$ & 0,001 \\
Cotos de fios adequados & $0,1(0,3)$ & $0,3(0,6)$ & $1,4(1,7)$ & $2,9(2,0)$ & $0,95(0,72 / 1,18)$ & 0,001 \\
Total de cotos de fios & $0,2(0,6)$ & $0,9(1,1)$ & $2,9(2,1)$ & $5,3(2,6)$ & $1,73(1,43 / 2,03)$ & 0,001 \\
Seminós iniciais ajustados & $0,2(0,4)$ & $0,7(0,7)$ & $1,8(1,0)$ & $2,9(1,4)$ & $0,92(0,76 / 1,07)$ & 0,001 \\
Total de seminós iniciais & $0,2(0,4)$ & $0,9(0,6)$ & $2,0(0,8)$ & $3,2(1,3)$ & $1,01(0,86 / 1,15)$ & 0,001 \\
Seminós seq. ajustados & $0,3(0,8)$ & $1,7(1,9)$ & $5,9(3,1)$ & $10,0(4,3)$ & $3,32(2,84 / 3,80)$ & 0,001 \\
Total de seminós seq. & $0,3(0,8)$ & $1,8(2,0)$ & $6,3(3,2)$ & $10,4(4,3)$ & $3,49(2,99 / 3,98)$ & 0,001 \\
\hline
\end{tabular}

-x:Média, DP:Desvio padrão, obed: obediência, não obed: não obediência, seq:sequenciais

Figura 2. Progressão na execução do número de pontos laparoscópicos por avaliação/etapa.

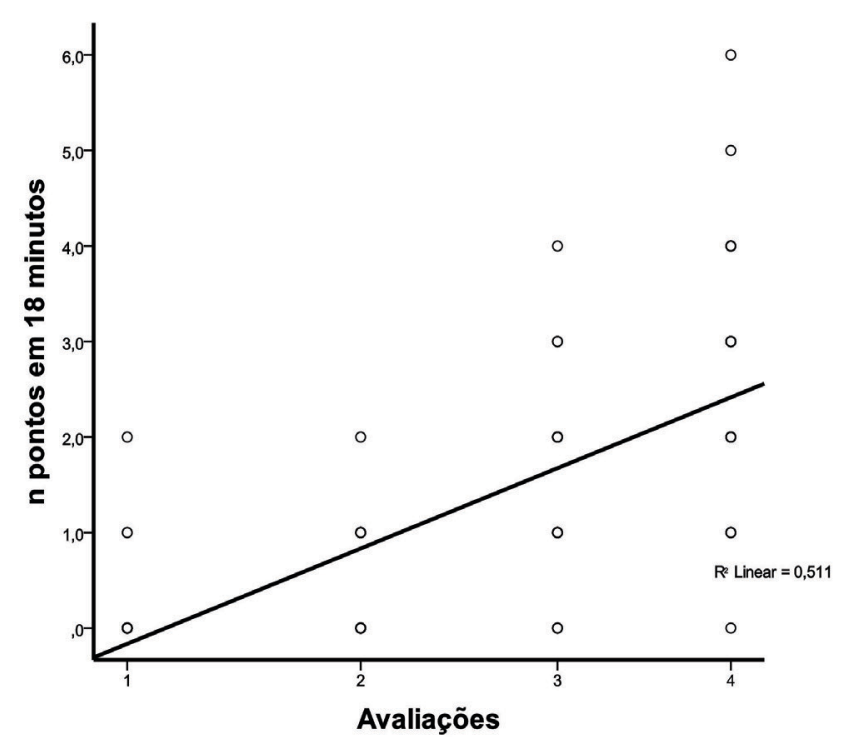

\section{DISCUSSÃO}

O resultado mais significativo da pesquisa foi o valor da média do número de pontos laparoscópicos na quarta etapa (avaliação final dos estudantes) do treinamento igual a 2,7. A comparação desse resultado com os de outras pesquisas evidenciou que a progressão da competência adquirida foi além do esperado.

Rosser et al. (1997) publicaram um modelo padronizado de treinamento para 150 cirurgiões, todos qualificados, com média de idade de 42,2 anos, no Departamento de Cirurgia da Universidade de Yale, Estados Unidos da América, com duração de três dias. Essa capacitação apresentava seis etapas de treinamento com dez secções de repetições em cada etapa. 0 tempo médio de execução de um nó intracorpóreo com quatro
Figura 3. Curva de aprendizagem dos estudantes de medicina na realização de pontos laparoscópicos por avaliação/etapa.

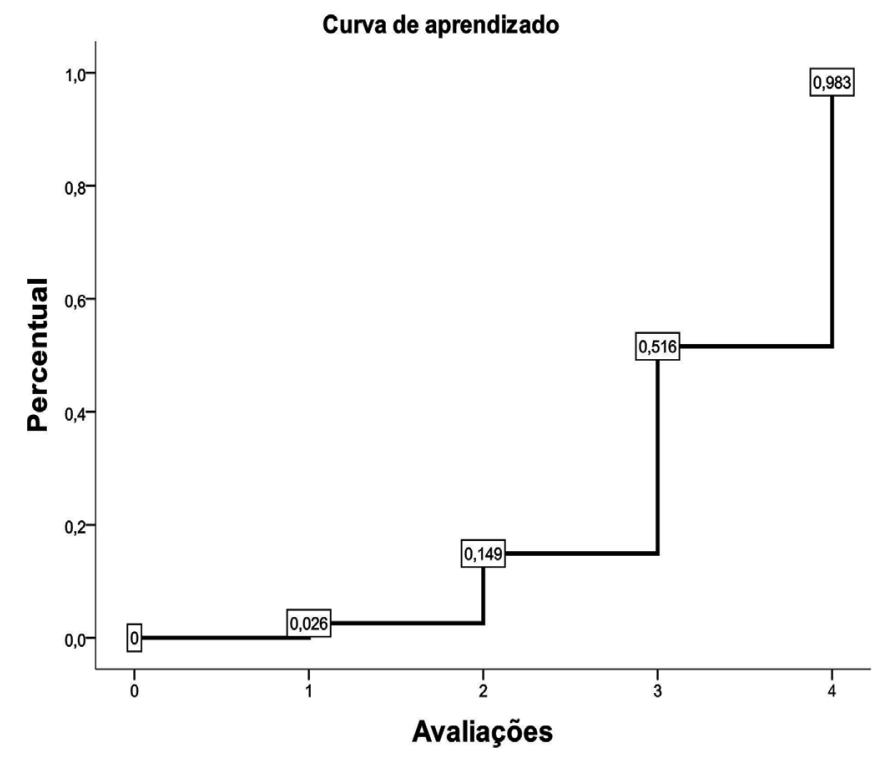

seminós antes do treinamento foi de seis minutos e dezesseis segundos ${ }^{21}$.

No modelo de treinamento aplicado na presente pesquisa, dos 29 alunos testados, o tempo médio de execução de um nó laparoscópico intracorpóreo com cinco seminós ao final do treinamento foi de seis minutos e 42 segundos. Os resultados obtidos pelos alunos treinados e que não tinham experiência prévia se equipararam aos dos cirurgiões antes do treinamento.

Depois do treinamento, os estudantes tornaram-se competentes na realização de seminós e nós em ambiente simulado de laparoscopia. A importância da aquisição da habilidade em 
realizar nós e pontos em laparoscopia para cirurgiões resulta no fato de que essa técnica cirúrgica é necessária para a realização de muitas operações laparoscópicas complexas. O não domínio dessa habilidade impede que os cirurgiões praticantes realizem procedimentos mais avançados ${ }^{24,25}$.

O único aluno que não conseguiu realizar pontos laparoscópicos ao final do treinamento demonstrou, na reunião final, uma indisfarçável frustração. Era evidente a dificuldade que ele demonstrava para realizar as manobras propostas. Fez algumas observações acerca do modelo de ensino do treinamento. Foi recomendado que, no momento em que estivessem treinando a realização de suturas na grade curricular do curso, ele aumentasse o número de repetições das tarefas.

Os nós cirúrgicos são definidos como o entrelaçamento entre as extremidades de um fio com o objetivo de uni-las ou fixálas. São comumente utilizados na aproximação de estruturas anatômicas ou ligaduras com finalidades hemostáticas ${ }^{26}$.

O nó é composto por um conjunto de seminós com as funções de contenção, fixação e segurança. Geralmente, são necessários três ou mais seminós para garantir a necessária segurança ${ }^{23}$. O ponto cirúrgico, segundo Magalhães, é a porção de fio compreendida entre os furos ou os locais de apoio realizados nos tecidos ${ }^{26}$.

A estruturação do modelo teórico prático de ensino seguiu os conceitos da Taxonomia de Dave, estabelecidos em 1967, e norteia a aquisição de habilidades psicomotoras ${ }^{27}$.

Algumas teorias educacionais deram suporte ao modelo de ensino: a teoria de Fitts e Posner, na qual o aprendizado ocorre por meio da aquisição e retenção da habilidade psicomotora, em três estágios sequenciais: cognição; associação e autonomia; a Teoria de Ericsons, cujo escopo é o desenvolvimento da habilidade após prática repetida do exercício, e a Teoria de Miller que propôs uma sequência hierárquica de competência em quatro níveis, tendo como base o "saber", seguido do "saber como", "demonstrar como" e, finalmente, o "fazer". Dessa forma, estabeleceu o passo a passo para a competência, no qual o aprendiz avança por meio dos passos cognitivos e comportamentais necessários que estão subjacentes ao próximo passo. E, por fim, o feedback, que é, sabidamente, uma das estratégias educacionais e avaliativas mais eficaz ${ }^{19}$.

A disposição sequencial do modelo de treinamento foi, em parte, embasada no trabalho de Dawidek e col, que apresentou evidências de que o treinamento incorporando a direta visualização, três dimensões, antes da visão indireta em duas dimensões, na simulação laparoscópica, acelerava a aquisição das habilidades ${ }^{14}$.

Dehabadi e cols publicaram, em 2014, artigo de revisão acerca do uso de simuladores na aquisição de habilidades de sutura laparoscópica e concluíram que a simulação é uma ferramenta útil no treinamento de futuros cirurgiões laparoscópicos, entretanto sugeriram que mais pesquisas seriam necessárias para responder à questão de como maximizar esse benefício28. Estudos observacionais indicaram que nós e suturas laparoscópicas podem ser aprendidos com sucesso por residentes de cirurgia em cursos de 1 a 5 dias, usando simuladores, e demonstraram que tanto estudantes sem experiência em laparoscopia, como estagiários com experiência laparoscópica anterior se beneficiam, significativamente, de tais $\operatorname{cursos}^{9,29,30}$. Além disso, descobriu-se que quase $73 \%$ dos participantes relataram, posteriomente, ser capazes de aplicar suas habilidades de sutura laparoscópica em situações reais ${ }^{31}$.

Uma publicação do Departamento de Cirurgia da Universidade de Manitoba, Canadá, que avaliava o desempenho de residentes de cirurgia geral e urologia, demonstrou que nem o simulador de baixa fidelidade nem o simulador virtual de alta fidelidade apresentavam correlação significativa com os objetivos do Global Operative Assessment of Laparoscopic Skills (GOALS). Esses resultados sugerem cautela com o uso dessas modalidades para avaliações de cirurgiões novatos e para processos de seleção ${ }^{15}$.

Sabe-se que ainda não há consenso acerca do modelo de treinamento uma vez que as curvas de aprendizagem em cirurgia minimamente invasiva são longas. Em vista disso, em 2016, Hendrie e cols compararam o treinamento sequencial com o simultâneo das habilidades psicomotoras e visuais espaciais para a realização de nós e suturas treinadas em simuladores e concluíram que o sequencial foi mais rápido no início da curva de aprendizagem, porém não reduziu o tempo de treinamento total necessário para alcançar a proficiência ${ }^{32}$.

Ficou evidente, nos resultados da avaliação inicial, que os estudantes não tinham quaisquer habilidades prévias, o que sugere que o modelo do treinamento, de fato, foi relevante para a aprendizagem.

Entretanto, precisa-se de mais pesquisas para responder se as habilidades adquiridas são compatíveis com as de cirurgiões já experientes e ainda como essa experiência pode ser aplicada ao treinamento de residentes de cirurgia.

Como limitações da pesquisa, deve-se inferir que o trabalho com seres humanos por si pode gerar vieses quanto ao aprendizado, visto que não é possivel garantir que os alunos, após o final de cada etapa do treinamento, não decidam, por conta própria, realizar algum exercício que possa interferir no resultado final, ou ainda que visualizem cirurgias em cenários reais e vídeos que possam, de certa forma, facilitar o aprendizado das manobras cirúrgicas do treinamento.

\section{CONCLUSÃO}

Os resultados da pesquisa, dentro de seu desenho próprio, mostram que os alunos submetidos ao modelo de treinamento sistematizado apresentaram progressão da competência ao final e entre as diversas etapas do treinamento, na realização 
de nós e pontos cirúrgicos em ambiente de simulação de laparoscopia. A curva de aprendizagem para realização de um ponto laparoscópico foi alcançada por quase todos os alunos.

Pesquisa realizada no laboratório de habilidades do Curso de Medicina do Centro Universitário Christus, Fortaleza,
Ceará, Brasil. A pesquisa foi financiada, parcialmente, pelo Centro Universitário Christus, motivo pelo qual agradecemos imensamente a seu corpo docente, discente e diretivo. Parte da tese de doutorado do Programa de Pós-graduação Stricto Sensu em Ciências Médico-Cirúrgicas do Departamento de Cirurgia da Faculdade de Medicina da Universidade Federal do Ceará.

\section{REFERÊNCIAS}

1. Davis SS Jr, Husain FA, Lin E, Nandipati KA, Perez S, Sweeney JF. Resident participation in index laparoscopic general surgical cases: impact of the learning environment on surgical outcomes. J Am Coll Surg. 2013 Jan;216(1):96-104. doi: 10.1016/j.jamcollsurg.2012.08.014.

2. Korndorffer JR Jr, Dunne JB, Sierra R, Stefanidis D, Touchard CL, Scott DJ. Simulator training for laparoscopic suture using performance goals translate to the operating room. J Am Coll Surg. 2005 Jul;201(1):23-9. doi: 10.1016/j. jamcollsurg.2005.02.021.

3. Peters JH, Freid GM, Swanstrom LL, Soper NJ, Sillin LF, Schirmer B, et al. Development and validation of a comprehensive program of education and assessment of the basic fundamentals of laparoscopic surgery. Surgery.2004; 135:21-27. doi: 10.1016/\$0039.

4. Moorthy K, Munz Y, Dosis A, Bello F, Chang A, Darzi A. Bimodal assessment of laparoscopic suturing skills: construct and concurrent validity. SurgEndosc. 2004 Nov;18(11):1608-12. doi: 10.1007/s00464-003-9312-5.

5. Rodrigues SP, Horeman T, Blomjous MS, Hiemstra E, van den Dobbelsteen $\mathrm{JJ}$, Jansen FW. Laparoscopic suturing learning curve in an open versus closed box trainer. Surg. Endosc. 2016; 30(1):315-22. doi: 10.1007/s00464-015-4211-0.

6. Alarcom A, Berguer R. A comparison of opening room crowding between open and laparoscopic operations. Surg Endosc. 1996 Sep;10(9):916-9.

7. Subramonian K, DeSylva S, Bishai P, Thompson P, Muir G. Acquiring surgical skills: a comparative study of open versus laparoscopic surgery. Eur Urol. 2004 Mar; 45(3): 346-351. doi: 10.1016/j.eururo.2003.09.021.

8. Cavalini WLP, Claus CMP, Dimbarre D, Cury AM Filho, Bonin EA, Loureiro MP, et al. Development of laparoscopic skills in medical students to surgical training. Einstein. 2014 Oct-Dez;12(4):467-72. doi: 10.1590/S1679-45082014AO3237.

9. Aggarwal R, Hance J, Undre S, Ratnasothy J, Moorthy K, Chang A, et al. Training junior operative residents in laparoscopic suturing skills is feasible and efficacious. Surgery. 2006 Jun;139(6):729-34. doi: https://doi.org/10.1016/j. surg.2005.12.010.

10. Hogle NJ, Briggs WM, Fowler DL. Documenting a learning curve and test-retest reliability of two tasks on a virtual reality training simulator in laparoscopic surgery. J Surg Educ. 2007 Nov-Dec; 64(6):424-30. doi: 10.1016/j. jsurg.2007.08.007.

11. Lents GM, Mandel LS, Lee D, Gardella C, Melville J, Goff BA. Testing surgical skills of obstetric and gynecology residents in a bench laboratory setting: validityan reliability. Am J Obstet Gynecol. 2001 Jun;184(7):1462-8.

12. Buzink S, Soltes M, Radonak J, Fingerhut A, Hanna G, Jakimowicz J. Laparoscopic Surgical Skills programme: preliminary evaluation of Grade I Leve 1 courses by trainees. Wideochir Inne Tech Maloinwazyjne; 7(3): 188-192. doi: 10.5114/wiitm.2011.28895

13. Roberts KE, Bell RL, Duffy AJ. Evolution of the skill training. World J Gastroenterol. 2006 May;12(20): 3219-24. doi: 10.3748/wjg.v12.i20.3219.

14. Dawidek MT, Roach VA, Ott MC, Wilson TD. Changing the learning curve in novice laparoscopists: incorporating direct visualization into the simulation training program. J Surg Educ. 2017 Jan-Feb;74(1):30-6. doi: 10.1016/j. jsurg.2016.07.012.
15. Steigerwald SN, Park J, Hardy KM, Gillman L, Vergis AS. The Fundamentals of Laparoscopic Surgery and LapVR evaluation metrics may not correlate with operative performance in a novice cohort. Med Educ Online. 20. doi: 10.3402/ meo.v20.30024.

16. Medeiros AC. Laboratório experimental. In: Saad R Jr, Salles RARV, Carvalho WR, Maia AM, editores. Tratado de Cirurgia do CBC. São Paulo: Atheneu; 2012. p. $1507-12$.

17. Nácul MP, Cavazzola LT, Melo MC. Situação atual do treinamento de médicos residentes em videocirurgia no Brasil: uma análise crítica. $A B C D$, arq bras cir dig. 2015; 28(1): 81-5

18. Schilickum M, Hedman L, Enochson L, Henningsohn L, Kjellin A, FellanderTsai L. Surgical simulation tasks challenge visual working memory and visualspatial memory ability differently. World J Surg. 2011 Apr; 35(4):710-5. doi: 10.1007/s00268-011-0981-2

19. Sadideen $\mathrm{H}$, Kneebon R. Pratical skills teaching in contemporary surgical education: how can educational theory applied to promote effective learning? Am J Surg. 2012 Sep; 204(3): 396-401. doi: 10.1016/j.amjsurg.2011.12.020.

20. Farquharson AL, Cresswell AC, Beard JD, Chan P. Randomized trial of the effect of video feedback on the acquisition of surgical skills. Br J Surg. 2013 Oct; 100(11): 1448-53. doi: 10.1002/bjs.9237.

21. Rosser JC, Rosser, L E, Savalgi R S. Skill acquisition and assessment for laparoscopic surgery. Archives of Surgery, 1997 Feb; 132(2): 200-204. doi:10.1001/archsurg.1997.01430260098021.

22. Sedlack JD, Willians VM, Desimone J, Page D, Ghosh BC. Laparoscopic knot security. Surgery Laparosc Endosc. 1996; 6(2):144-146.

23. Marques, RG. Nós cirúrgicos. In: Marques, RG. Técnica operatória e cirurgia experimental. Rio de Janeiro: Guanabara Koogan; 2005. p. 315-24.

24. Harold KL, Matthews BD, Backus CL, Pratt BL, Heniford BT. Prospective randomized evaluation of surgical resident proficiency with laparoscopic suturing after course instruction. Surg Endosc. 2002 Dec;16(12):1729-31. doi: 0.1007/s00464-002-8832-8.

25. Nguyen NT, Mayer KL, Bold RJ, Larson M, Foster S, Ho HS, et al. Laparoscopic suturing evaluation among surgical residents. J Surg Res. 2000 Sep; 93(1):133-6. doi: 10.1006/jsre.2000.5969.

26. Bellen BV, Magalhães HP. Nós Cirúrgicos. In: Magalhães HP. Técnica cirúrgica e cirurgia experimental. São Paulo: Savier; 1989. p.74-76.

27. Simpson EJ. The Classification of Educational Objectives in the Psychomotor Domain. Washington DC: Gryphon House; 1972.

28. Dehabadi M, Bimbi F, Berlingieri P. The use of simulation in the acquisition of laparoscopic suturing skills. Int J Surg. 2014;12(4):258-68. doi: 10.1016/j. ijsu.2014.01.022.

29. Mereu L, Carri G, Albis Florez ED, Cofelice V, Pontis A, et al. Three-step model course to teach intracorporeal laparoscopic suturing. J Laparoendosc Adv Surg Tech A. 2013 Jan; 23(1): 26-32. doi: 10.1089/lap.2012.0131.

30. Stefanidis D, Sierra R, Korndorffer JR Jr, Dunne JB, Markley S, Touchard CL, et 
al. Intensive continuing medical education course training on simulators results in proficiency for laparoscopic suturing. Am J Surg. 2006 Jan;191(1): 23-7. doi: 10.1016/j.amjsurg.2005.06.046.

31. Rinewalt D, Du H, Velasco JM. Evaluation of a novel laparoscopic simulation laboratory curriculum. Surgery. 2012 Oct;152(4): 550-6. doi: 10.1016/j. surg.2012.08.009.

32. Hendrie JD, Nickel F, Bruckner T, Kowalewski KF, Garrow CR, Mantel M, et al. Sequential learning of psychomotor and visuospatial skills for laparoscopic suturing and knot tying-study protocol for a randomized controlled trial "The shoebox study". Trials. 2016; 17(14). 